\title{
Research on Management Model of Automobile Manufacturing Line Based on RFID Technology
}

\author{
Wan Teng ${ }^{1}$, Xiaoping Wang ${ }^{2}$ \\ ${ }^{1}$ School of Machinery Engineering, Baicheng Normal University, Baicheng, 137000, China \\ ${ }^{2}$ Taonan First Middle School, Taonan, 137100, China
}

Keywords: Automobile manufacturing Line, RFID technology, Management model

\begin{abstract}
RFID (Radio Frequency Identification) is a non-contact automatic recognition technology, which automatically identifies target objects and obtains relevant data through radio frequency signals. The identification work does not require manual intervention, and can work in a variety of harsh environments. This paper analyzes the important functions of RFID in automobile production line, and expounds the management models of coating, welding and assembly line based on RFID technology to provide some references for relative researchers.
\end{abstract}

\section{Introduction}

In 1990s, RFID began to rise as an automatic identification technology [1]. It attracted the attention and application from all walks of life with the advantages of data traceability and storage capacity. Radio frequency identification technology belongs to the process of realizing information recognition using space coupling radio frequency signals to reach the goal of non-contact transmission of information. The RFID system is composed of application software, antenna, reader and writer, and RF tag. Many domestic automobile companies use bar code to collect corresponding production line information, but this way has some shortcomings, if there is a large restriction in the production line. The amount of information that a bar code can carry is very limited. To read the corresponding information, you must guarantee the alignment of the scanner. If the bar code is shedding, fouling or tearing, it will be difficult to achieve the effect of scanning. In actual production, a lot of manpower needs to be invested, so it is easy to see the problem of mistake and leakage. And the use of RFID technology can be a perfect solution to the corresponding problems. RFID can memorize many data letters. She can communicate the information in a non - contact way and recognize it effectively. RF tags can be used in the dust and high temperature environment, the actual life is longer, and can be recycled. In actual use, the whole process can be automatically controlled. Radio frequency identification is an advanced recognition and acquisition technology, which can provide necessary data information for manufacturing execution system in time, and help management to make necessary production scheduling and plan adjustment, to improve the level of production plan execution. With the increasing opening of the automobile industry, the domestic automobile manufacturing industry is facing huge pressure of operation. At the same time, with the production and use of cars in large quantities, the demand for the development of the automobile industry will be higher and higher. All companies want to be able to establish an identification system to improve their management level to improve efficiency. Radio frequency identification technology can locate the location of a specific vehicle in the production line at random, and fully understand the actual completion progress and assembly [2].

\section{Functions of RFID Technology in Automobile Manufacturing Line}

\subsection{Collect Queue Information}

The RFID reader reads the VIN code information in the tag, and passes it to the background 
control system through the wireless network. The system automatically generates the queue information of the vehicle on the transport chain according to the VIN code of the vehicle. In case of emergency, the site operator can read the VIN code in the vehicle tag through a handheld data terminal to ensure the correctness of the production queue information. In this way, the timely acquisition of vehicle queue information can ensure the rational allocation of resources in the production site, and even in all kinds of emergencies, we can get the best resource scheduling plan in a relatively short time. In the automobile assembly workshop, the parts of the spraying body buffer need to be hoisted to the corresponding production line according to the corresponding plan. Because the torsional capacity of the sprayed body buffer is limited, it may be difficult to match the specific production plan. If RFID is used to collect the corresponding data, the label of the sprayed vehicle body buffer can be set at the top of the relevant vehicle, and the identification code of the representative vehicle can be entered. If the corresponding vehicle to the specific acquisition point, RFID reader can immediately read relevant tags in the VIN code information, and then use the wireless network transmission to the control system of production line, production line system of vehicles using the VIN code, can generate the corresponding letter message queue. If an emergency occurs, such as the need to cancel a vehicle to be installed or insert a temporary vehicle to be installed. Operators can use mobile data terminal to read information in specific tags, and manually modify the corresponding content, and ensure the accuracy of production queue information. Through this method, we can collect vehicle queue information effectively, and rationally allocate all aspects of the field resources. Even if we find an emergency, we can quickly provide effective resource scheduling strategy [3].

\subsection{Precise Track Vehicles}

In a mixed flow production line, it is necessary to track and locate vehicles in a timely manner. It is necessary to track the vehicle from the welding workshop, then pass the body in white buffer area, then go through the painting workshop, then go through the PBS area, and finally enter the final assembly shop to finish the inspection. Therefore, the use of RFID technology can effectively track and locate vehicles, and set up vehicle information collection points in the key parts. In the assembly shop, the radio frequency identification information collection point should be set up in the key position of the vehicle's downline point and the line point. If the vehicle passes the location information collection point, we can use the reader to scan the information in the tag, and use the database to get the corresponding vehicle location, and understand the actual vehicle assembly schedule. If the vehicle reaches the corresponding information collection point and gets the exact engine number of the corresponding vehicle, the on-site staff can match the actual VIN number and engine number, and then the corresponding tags can be recovered, and the cycle will be used. Through the summary of each vehicle assembly progress information, the assembly line of the whole assembly line can be obtained. When the vehicle assembly completes to the offline information collection point, the reader reads the VIN code information in the tag, and then supports the database to get the engine number of the vehicle through the database. At present, automotive flexible automatic welding production line is mainly the multi vehicle mixed production mode, which can improve the production management level of multi vehicle mixed production. Information acquisition, information processing, information transmission and control and tracking of target products in the production process become more and more important. Because the RFID technology has the advantages of non-contact read-write, high accuracy, high reliability, durability and environmental advantages, RFID technology management and the existing production line execution system combination, can provide fast and reliable information for the execution system, improve the management level of welding production line [4].

\subsection{Material Dynamic Distribution}

The dynamic distribution management of production line refers to the material distribution from workshop temporary storage area to line edge. After introducing RFID technology, the way of material delivery is also transformed from traditional static to dynamic. The material dynamic 
distribution, the actual time must consider the production rhythm of the production line, and the line side inventory preparation, feeding required to match, to plan for the real-time dynamic material. We use the RFID technology to obtain the information queue production line, to track the production schedule and production status of the online vehicle, the inferred real-time consumption and station WIP material production line material is sent to the material name, line number, material suppliers and other information placed in the box in the high frequency phase label bound advance side before, for online production timely collection of corresponding material consumption information. While in the automobile production line into a vehicle assembly area, fixed device to automatically read vehicle UHF tags in the VIN code to read and write the information on the PC station, station of the assembly information, the assembly personnel in accordance with the assembly information before taking the material, through the high frequency HF label handheld reader scan preset in the material box, the material information tags within the distribution system to dynamic materials, the system automatically cut the line station material inventory, when it is below a certain value when the Fed issued safety instructions, to ensure the normal supply of production material. We try to establish a dynamic real-time detection system of machine vision, follow the car body to take photos synchronously, and automatically detect the defects on the part of the vehicle surface. At the same time, we analyze the picture and the feedback data, supervise and adjust the quality of the welding in time.

\section{Applications of RFID Technology in Management Models of Automobile Manufacturing Line}

\subsection{Management Model of Coating Line Based on RFID Technology}

The process of automobile painting production line is complex, vehicle type changes and production flexibility are large, and the purpose of automobile painting is to make every car sold to make consumers look perfect. Therefore, the high-quality monitoring system and the level of automation are necessary for each process of the automobile painting line. The application of RFID technology in vehicle body recognition system of automobile painting production line, real-time collection of information in the coating production line, monitoring quality and statistical field data, ensures the timely collection of information in painting production control center. Skid conveyor is conveying equipment of painting production line, the high temperature resistance of passive RFID tags is installed in each carrying a body skid, the passive tag in the coating production process with the workpiece, as the body of mobile data to realize each body carry data. Aiming at different painting production management and technology, the application of RFID technology has set up a reading and writing station on the production site, such as entry of the important process in the workshop, drying room, spray paint room and storage area, etc. These locales reading and writing stations can write the body information, the number of spraying, the color and the skid, and deliver the information to the control center of the painting line. The application of RFID technology improves the automation and flexibility of the auto painting production, reduces the error rate of the system and improves the production efficiency. Therefore, every process link of the workshop production line must have a very high level of automation and quality monitoring system. We can collect all kinds of on-site data, such as statistics, status monitoring and quality control, and send them to production control center in time.

\subsection{Management Model of Welding Line Based on RFID Technology}

In the automobile production line, RFID technology can play its anti-dust, anti-magnetic, anti-metal, can be reused and the characteristics of high temperature, the monitoring of welding production line through the automatic recognition work, can be the kind of car, and the welding procedure information indicating information indicating information stored in the target tray the antenna is mounted in the welding production line, the high places, and people can avoid the influence of machine. RFID tags with strong durability and strong pollution resistance can effectively 
improve the visibility of the internal supply chain, and greatly reduce the recognition error and human error rate in the actual automobile welding production line. In the early automobile body welding line, most of the manual methods are used to see the written operation instructions step by step, and it is easy to make human error. Later, it gradually changed to bar code label management. Although it reduced human error rate, it often made mistakes due to the influence of dust, high temperature vibration and oil pollution at the production site. To meet with a situation, whether the barcode label manual and ease of use. To this end, the use of REID technology can achieve good welding management effect. We choose high temperature, dust protection, metal protection, magnetic field and reusable active packaging labels to monitor the operation of welding production line through automatic identification operation. The operating instructions to the letter car information, welding procedure, indication information stored in the target tray, the antenna to avoid the impact of human and machine can be set in the welding production line in high places, to RFID tag strong anti-pollution ability and strong durability, greatly reduce the recognition error and can effectively improve the visual degree internal supply chain.

\subsection{Management Model of Assembly Line Based on RFID Technology}

The implementation of traditional car assembly progress monitoring and tracking is done manually in a paper-based identification card or bar code. The paper identification card and bar code are prone to loss, damage or replace the phenomenon that results in the actual production in automobile assembly error problem. The application of RFID technology in the automobile assembly line in synchronized assembly process, especially used in the production line of JIT production mode, so that the parts and materials sent to the station on time and improve the material supply and inventory, and assemble into vehicles of organic complexes. The reusable and Recyclable RFID tags installed on the rack assembly of the vehicle, making each assembly of vehicle number, and the reader will write the RFID tag reader, and will be installed in each suitable operating point, reduces the error rate of assembly line. The application of RFID technology has the characteristics of real-time monitoring of production line quality monitoring and production data, and realizes the production scheduling, raw material supply and quality monitoring and tracking in the total vehicle production line, which greatly reduces the production error rate. With the continuous development of RFID technology and the popularization and application of application system, the application of RFID technology will improve greatly in terms of performance, and the cost will also gradually decrease. RFID technology will certainly become the mainstream of automobile information manufacturing and automotive information management, thus promoting the development of the automobile industry. On the assembly line, especially in the line of JIT production, raw materials and components must be sent to workstations on time. Inventory and material supply must also cooperate with vehicle assembly progress. Before using RFID technology, automobile factory is to manually by using bar code or paper identification card to realize real-time tracking and monitoring of the vehicle assembly schedule, it is extremely easy to be destroyed or lost, replace the bar code and identification card, causing the production operation error operation. Therefore, the automobile industry is trying to use RFID technology to synchronize the process target.

\section{Conclusion}

The application of RFID technology in the automobile production line has the advantages unparalleled by other technologies. This paper discusses the specific application of RFID, and discusses the system of automobile manufacturing production line based on RFID technology. RFID technology will certainly become the mainstream of automobile information manufacturing and automotive information management, thus promoting the development of the automobile industry.

\section{References}

[1] Zhou Guodong, Zhang DunLi. The Application of Siemens Ethernet RFID Solutions on the 
Design for the Communication Management Program [J]. Journal of Jiamusi University (Natural Science Edition), 2014, 32(1): 88-91.

[2] Jiang Ru, Zhang Geyan, Song Hongman, et al. Configuration method of Balluff RFID technology in Siemens PLC in auto production line [J]. Automobile Applied Technology, 2015(12): 132-133.

[3] Li Mu, Kong Shuhua. Application on automotive welding line and analysis of influence factors [J]. Electric Welding Machine, 2013, 43(6): 35-38.

[4] Zhong Yuanchang, Sun Lijing, Ding Xuan, et al. Application actuality of RFID in the automobile production line [J]. Microcomputer \& Its Applications, 2010(3): 1-3+7. 